\title{
Evaluation of Health Concern using Big Facts Analytics
}

\author{
K. Anita Davamani, S.Amudha, G.Michael, B.Sundarraj
}

\begin{abstract}
This paper gives a concise presentation about how we can reveal extra an incentive from wellbeing data utilized in human services focuses utilizing another data the board approach called as large information investigation .Including huge information examination in wellbeing area gives partners new bits of knowledge that can possibly progress customized care, enhance persistent results and keep away from superfluous expenses. This paper characterizes huge information examination and its qualities, remarks on its points of interest and difficulties in human services.
\end{abstract}

Keywords:Datascreening,big data,health care.

\section{I.INTRODUCTION}

The social insurance enterprise truly has produced lots of statistics, pushed by means of file retaining, consistence and administrative requirements, and affected person attention. While most facts is placed away in published replica shape, the present pattern is toward fast digitization of those loads of information. Driven via compulsory necessities and the opportunity to beautify the character of human services conveyance within the period in-between diminishing the fees, these big amounts of records (referred to as 'large statistics') hold the assure of helping a extensive scope of healing and social insurance capacities, to infer already undiscovered knowledge and bits of knowledge from information to address numerous new and essential inquiries. Inside the wellbeing segment, it furnishes partners with new bits of knowledge that can possibly progress customized care, enhance tolerant results and maintain a strategic distance from pointless expenses.

By definition, huge statistics in medicinal offerings alludes to electronic wellbeing informational indexes so sizeable and complex that they may be difficult (or incomprehensible) to deal with commonplace programming or probably device; nor could they be able to be efficiently made do with conventional or everyday statistics the executives contraptions and techniques. Enormous data in human offerings is overpowering a end result of its volume in

Revised Manuscript Received on June 22, 2019.

K.Anitha Davamani, Depaetment of CSE, Bharath Institute of Higher Education and Research,Chennai, ThamilNadu, India.

S.Amudha ,Department of CSE,,Bharath Institute of Higher Education and Research,Chennai,Thamilnadu,India.

G.Michael, Depaetment of CSE, Bharath Institute of Higher Education and Research, Chennai, ThamilNadu, India.

B.Sundarraj ,Department of CSE,,Bharath Institute of Higher Education and Research,Chennai,Thamilnadu,India. addition to as a consequence of the decent form of facts sorts and the rate at which it must be overseen.

It incorporates clinical facts and medical preference emotionally supportive networks (health practitioner's composed notes and treatments, restorative imaging, lab, drug save, safety, and different regulatory statistics); quiet statistics in digital affected person information (EPRs); gadget produced/sensor information, for instance, from checking important signs; internet primarily based lifestyles posts, including Twitter channels (supposed tweets), web sites, notices on Facebook and unique levels, and site pages; and much less patient-specific information, which include crisis care statistics, news assets, and articles in healing diary.

There may be chance for the significant facts scientist, among this amount of the first rate and show off of data. By discovering affiliations and understanding examples and styles within the information, good size data review may likely improve care, spare lives, and reduced prices. As a result, enormous data study packages in medicinal facilities take benefit of the blast in statistics..

Investigation when connected with regards to huge information is the way toward inspecting a lot of

information, from an assortment of information sources and in various organizations, to convey bits of knowledge that can empower choices in genuine or close constant. Different explanatory ideas, for example, information mining, characteristic dialect handling, man-made consciousness and prescient examination can be utilized to dissect, contextualize and picture the information.

Huge information logical methodologies can be utilized to perceive intrinsic examples, connections and peculiarities which can be found because of incorporating huge measures of information from various informational indexes.

This article provides a review of enormous human services data inquiry as it is growing as a control. To begin with, we characterize and examine the significance of vast data and characteristics of vast inquiry of data in medicinal services. At that stage we portray the types of huge social insurance data. Third, we offer cases of enormous inquiry of data

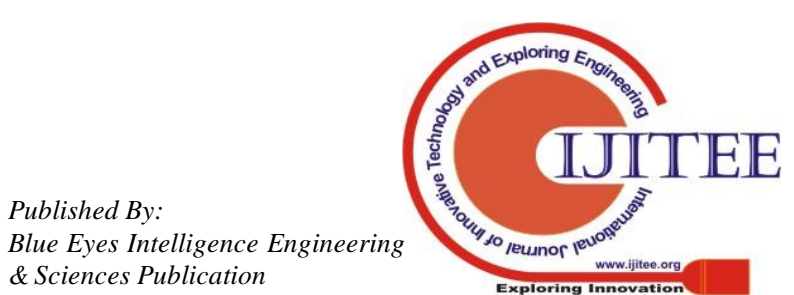




\section{DEFINING BIG DATA.}

Enormous information commonly alludes to the accompanying kinds of information:

-Traditional challenge information - incorporates customer statistics from CRM frameworks, value-based ERP information, web keep exchanges, and popular report records.

-Machine-produced/sensor statistics - includes Call Detail Records ("CDR"), weblogs, sensible meters, fabricating sensors, hardware logs (frequently alluded to as superior fumes), and changing frameworks facts.

-Social statistics - contains patron enter streams, small scale blogging locales like Twitter, online networking levels like Facebook

Truth be informed, there are 4 key attributes that signify huge statistics:

-Volume is the measure of information created by using institutions or people. Ventures in all corporations are searching for methods to address the regularly increasing information volume this is being made each day.

-Velocity is the recurrence and speed at which data is produced, captured and communicated. Customers as well as businesses are currently producing higher statistics and lots of shorter cycles, from hours, minutes, seconds down to milliseconds.

-Variety is the expansion of new facts types consisting of those from social, machine and transportable assets. New kinds comprise substance, location or geo-spatial, device statistics focuses, $\log$ facts, system data, measurements, versatile, physical statistics focuses, method, radio recurrence distinguishing evidence (RFID), appearance, feeling, spilling records, social, content material and net. Likewise, collection carries customary unstructured medical records (i.E., loose content).

\section{BIG DATA'S ROLE IN HEALTH CARE}

\section{A.The varieties of facts foreseen to be useful in BDA consist of:}

1.Clinical information - as much as 80 percent of wellbeing statistics is unstructured as reviews, photographs, scientific or advocated notes;

2.Publications - clinical research and medicinal reference cloth;

3.Clinical references - content based totally exercise guidelines and wellbeing item (e.G., sedate records) information;

4.Genomic statistics - speaks to large measures of recent quality sequencing statistics;
5.Streamed records - home staring at, telehealth, handheld and sensor-based far off or sensible devices are new facts resources and types;

6.Web and lengthy variety interpersonal communique facts consumer utilization of Internet - facts from internet crawlers and individual to character conversation destinations; and

7.Business, hierarchical and outer information - managerial information, for example, charging and planning and other non-wellbeing information.

\section{IV.WAYS OF BDA IN HEALTH CARE}

Huge statistics research speaks to some other manner to address exam. It doesn't yet have an expansive or important influence India or globally. Be that as it can, the proceeding with digitization of wellness records collectively with the interoperable electronic health file (EHR), displays new probabilities to explore a horde of scientific and authoritative inquiries.

There is potential to layer BDA-kind programs, in a protection protective manner, over the number one wellbeing IT basis to determine esteem that may not usually be located. What pursues are some innovative thoughts and preparations.

- Clinical preference assist - BDA advancements that filter out via a whole lot of information, realize, order and gain from it, and afterward anticipate effects or prescribe non-obligatory medicines to clinicians and sufferers at the reason of attention.

-Personalized care-Predictive data mining or systematic preparations that can use tailored care (e.g., genomic DNA grouping for malignancy care) to frequently feature enjoyable exercise medicines for patients. These preparations may also give early discovery and assessment before a person generates the outcomes of the facet of illness.

-Public and populace health - BDA arrangements which can mine digital and net primarily based lifestyles statistics to anticipate influenza flare-americadependent on buyers' hunt, social substance and question movement. BDA arrangements can likewise bolster clinicians and ailment transmission specialists appearing investigations crosswise over patient populaces and care scenes to assist distinguish disorder styles.

- Clinical sports - BDA can bolster activities, as an instance, maintain up time the board, in which it may mine quite a few recorded and unstructured information, search for examples and model distinct situations to foresee activities which can affect keep up times before they really occur.

-Policy, budgetary and managerial - BDA can bolster leaders by means of incorporating and inspecting facts identified with key execution markers. 


\section{IMPLEMENTATION PROGRESS OF BDA WITHIN THE HEALTH CARE SECTORS.}

Example1: Disease episode BDA applied to look at the organization of ailments. Google is a variant. The use of BDA by the organization to contemplate the scheduling and proximity of internet crawler requests to count on disease flare-ups. Research shows that 33 percent of clients are currently using long-range interpersonal discussion for medicinal offering reasons (Facebook, YouTube, websites, Google, Twitter). As demands

Example2:Question and respond - clinical choice help

Research demonstrates that 79 percent of supplier associations inside the U.S. Are swinging to medical informatics with an stop aim to anticipate therapeutic mistakes. Sixty one percent assume investigation to decorate populace well being, and 52 percent display that examination driven preventive attention will help get manage over expenses. BDA can likely assist enhance present paintings process and outcomes from business procedures, as an instance, arrangement handling, planning, e-referral and e-release.

BDA can likewise help with giving bits of information around holes in the continuum of attention crosswise over settings and function satisfactory practices in attention bureaucracy and clinical results.

Question and answer preparations coordinated with mechanized supplier arrange passage (CPOE), e-referral, e-release and exceptional system based sporting events can be applied to break down and expect slants in social insurance.

BDA can mine volumes of medicinal writing and other unstructured information and coordinate these results with the growing amounts of discrete documents captured in EHRs, EMRs and PHRs. BDA can strengthen content review, fully data-based proof and through function dialect getting ready innovation can recognize, examine and in a while expect destiny occasions. These exam are then recommended again to clinicians as contemplations in their basic management.

Patients or clients likewise use BDA to find answers for their own situations. Information can be exhibited clearly and empower know-how cooperation of their social insurance designs and conceivably decrease re-confirmations or unfavourable consequences.

Example3:Heritage health price Distinguish patients will's identity admitted to a healing facility inside the following year utilizing chronicled claims information. Over \$30 billion was spent on superfluous healing facility confirmations. Objectives: Recognize patients at high chance and ensure that they receive the remedy they need. Create calculations to predict the amount of days a individual will spend in a clinic in the following year.

\section{RESULTS:}

Human services providers can grow new strategies to reflect on consideration on sufferers previously its beyond the factor of no go back decreases the quantity of superfluous hospitalizations. Improving the power of sufferers at the same time as diminishing the prices of attention. Winning arrangements utilize a mixture of some prescient fashions.

Example4: Data streaming BDA preparations provide an approximate consistency of treatment and negative operations to clinicians and patients with early or prescient pieces of knowledge. The model Demonstrates the use of BDA at the Toronto Hospital for Sick Children to orchestrate downpour records that screen traps from neonates (for example, more than 1,000 accounts for every 2nd physiological estimate). The BDA agreement provides pieces of know-how that allow researchers to assume calculations when a little one has a opportunity of illness. As close to home wellness equipment.

Example5:Genomics and personalized care

Customized care is displayed as the subsequent rush of return in the conveyance of restorative treatment to sufferers. The biggest association of facts on human hereditary spectrum generated by the frequently occurring one thousand Genomes Project-is currently openly useful on the Amazon Web Services (AWS) cloud. At two hundred terabytes-the sixteen million file

Example6: Penalties for poor care - 30 days readmissions Hospitalizations represent over $30 \%$ of the 2 Trillion yearly price of social insurance within the United States. Around $20 \%$ of all medical institution confirmations show up interior 30 days of a beyond launch. Expensive as well as probably unfavourable, and mainlyThey are often preventable. Medicare punishes hospitals with elevated readmission rates among patients with coronary heart unhappiness, coronary heart attack, and pneumonia.

Recognizing patients at risk of readmission can handle efficient use of assets and can possibly spare a broad range of annual social insurance dollars.Correctly making expectations from such complicated hospitalization facts would require the improvement of novel progressed logical fashions.

Example7: Consumer based online life .This model includes utilization of on-line life and systems to endorse clients of situations and self-administration pointers. The model portrayed to 1 facet is the combination and provisioning of population based information inside lengthy variety interpersonal communique situations to greater without difficulty advise and attract sufferers in dealing with their health and to effect or change their propensities for conformance to remedy alternatives, plans and satisfactory practices.

Be that as it can, the usage of interpersonal company internet searcher toolboxs poses some exciting lawful or chance contemplations. Merchants of these tool boxs might not deliver proof based totally suggestions and treatment options to sufferers. Actually, sufferers might be in chance when they 
self-examine dependent on those proposals.

Example8:Supporting wellbeing advancement using open wellbeing information

The U.S. Open Health Data Initiative is an open private exertion that means to enable Americans to comprehend wellbeing and social insurance execution in their networks. This work fixates on catalyzing the appearance of a system of network wellbeing information providers (HHS) and "information appliers" who use that information to make BDA applications that bring issues to light of network wellbeing execution, increment weight on leaders to enhance execution, and help encourage and educate activity to enhance execution. Working with a developing exhibit of social insurance partners, they will look for distinguish the employments of this information that would do the most to bring issues to light of wellbeing execution, and help propel city pioneers and natives to enhance execution.

\section{B.Potential instances of employments include:}

Intuitive well-being maps at the web that allow nationals to comprehend wellness execution in their well known vicinity as opposed to extraordinary territories, without breaking a sweat and lucidity; "dashboards" that empower city hall leaders and other urban pioneers to follow and advertise neighborhood wellbeing execution and issues;

Person to person communication applications that permit wellbeing enhancement pioneers to associate with one another, look at execution, share best practices, and test one another; Rivalries with respect to how networks can enhance to enhance wellbeing execution; viral web based amusements that assistance instruct individuals about network wellbeing; usage of network wellbeing information to help enhance the convenience of results conveyed by web search tools when individuals do wellbeing related pursuits and further bring issues to light of network wellbeing execution; and joining of network wellbeing related information into new settings, for example, land sites, which could be exceptionally successful disseminators of such data.

\section{CHALLENGES ON HEALTH CARE.}

- Use of patient / fact correlations in longitudinal documents. - Understanding unstructured clinical notes in the correct context.

- Efficient handling of large amounts of clinical imaging data and likely the extraction of helpful data and biomarkers.

- Analyzing genomic stats is a computationally intensive task and blending with conventional science

\section{VIII.CONCLUSION AND FUTURE WORK}

Big information analytics in healthcare is evolving into a promising subject for supplying insight from very huge statistics sets and improving effects at the same time as lowering fees. Its potential is incredible; but there continue to be demanding situations to conquer Big facts analytics has the ability to transform the way healthcare businesses use state-of - the-art techniques to obtain understanding from their repositories of science and various facts and create informed choices. In the destiny, we will see the rapid, sizable implementation and use of large-scale information.

\section{REFERENCES}

1. Dembosky A: "Data Prescription for Better Healthcare." Financial Times, December 12, 2012,

2. Feldman B, Martin EM, Skotnes T:"Big Data in Healthcare Hype and Hope." October 2012. Dr. Bonnie.

3. Fernandes L, O'Connor M, Weaver V: Big data, bigger outcomes.J AHIMA 2012, 38-42.

4. Transforming Health Care through Big Data Strategies for leveraging big data in the health care industry. 2013.

5. Wullianallur Raghupathi and Viju Raghupathi Big data analytics in healthcare: promise and potential

6. Suthaharan, Shan. "Big data classification: Problems and challenges in network intrusion prediction with machine learning." ACM SIGMETRICS Performance Evaluation Review 41.4 (2014): 70-73.

7. RAO, BLS PRAKASA. "of the Notes: Brief Notes on BIG DATA: A Cursory Look." (2015).

8. Raghupathi, Wullianallur, and Viju Raghupathi. "Big data analytics in healthcare: promise and potential." Health Information Science and Systems 2.1 (2014): 3.

9. Fang, Ong Huey, Norwati Mustapha, and Md Nasir Sulaiman. "Integrating biological information for feature selection in microarray data classification." Computer Engineering and Applications (ICCEA), 2010 Second International Conference on. Vol. 2. IEEE, 2010.

10. Hermon, Rebecca, and Patricia AH Williams. "Big data in healthcare: What is it used for?." (2014).

11. Groves, Peter, et al. "The 'big data'revolution in healthcare." McKinsey Quarterly (2013).

12. Rama Satish, K. V., and N. P. Kavya. "Big data processing with harnessing hadoop-MapReduce for optimizing analytical workloads." Contemporary Computing and Informatics (IC3I), 2014 International Conference on. IEEE, 2014.

13. Er, Orhan, Nejat Yumusak, and Feyzullah Temurtas. "Chest diseases diagnosis using artificial neural networks." Expert Systems with Applications 37.12 (2010): 7648-7655.

14. Jen, Chih-Hung, et al. "Application of classification techniques on development an early-warning system for chronic illnesses." Expert Systems with Applications 39.10 (2012): 8852-8858.

15. Yu, Lei, and Huan Liu. "Feature selection for high-dimensional data: A fast correlation-based filter solution." ICML. Vol. 3. 2003.

16. Mall, Raghvendra, et al. "Representative subsets for big data learning using k-NN graphs." Big Data (Big Data), 2014 IEEE International Conference on. IEEE, 2014. 


\title{
AUTHORS PROFILE
}

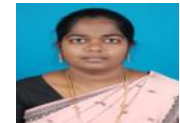

K.Anitha Davamani, Depaetment of CSE, Bharath Institute of Higher Education and Research ,Chennai, ThamilNadu, India.

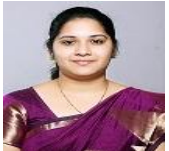

\begin{abstract}
S.Amudha ,Department of CSE,,Bharath Institute of Higher Education and
\end{abstract} Research,Chennai,Thamilnadu,India.

G.Michael, Associate Professor,Department of CSE, Bharath Institute of Higher Education and Research, Chennai, Tamilnadu, India.

B.Sundarraj, Assistant Professor, Department of Computer Science \& Engineering, Bharath Institute of Higher Education and Research, Chennai, India 